\title{
Cytomorphological Studies in the Tetrasomics of Egyptian Henbane, Hyoscyamus muticus $L$.
}

\author{
J. R. Bahl and B. R. Tyagi \\ Central Institute of Medicinal and Aromatic Plants, \\ Post Bag No. 1, R.S.M. Nagar, Lucknow 226016, India
}

Accepted March 22, 1992

The plants with an extra dose of a pair of homologous chromosomes in the euploid complement $(2 n+2)$ are known as tetrasomics. Such aneuploids have been reported in different plant species like Datura (Blakeslee and Belling 1924), Zea (McClintok 1929), Nicotiana (Goodspeed and Avery 1939), alfalfa (Kasha and McLennan 1967), Hordeum (Tsuchiya 1962, 1967, 1969), tomato (Rick and Gill 1973), Oryza sativa (Khush et al. 1984) Coix gigantea (Sapre and Barve 1987) and Plantago lagopus (Bhan et al. 1990). Tetrasomics generally appear in the progeny of autotriploids and primary trisomics, they are also likely to originate from diploids and monosomics (Khush 1973).

Hyoscyamus muticus L. $(2 n=28)$ of the family Solanaceae is an important commercial source of pharmaceutically valuable tropane alkaloid-hyoscyamine (Grieve 1977). In our on-going programme aimed at establishing a complete set of primary trisomics for use in gene mapping in $H$. muticus, autotriploids have been produced in an inbred CIMAP/NP-41 developed at this Institute (Tyagi 1986) and subsequently studied for their meiotic and breeding behaviour (Tyagi and Dubey 1989a, Tyagi 1990). During the study of breeding behaviour of an autotriploid of $H$. muticus, two tetrasomics were identified. The morphological and cytological features of these tetrasomics are described in this paper.

\section{Materials and methods}

The two tetrasomic plants (TS-1 and TS-2) used in this study were obtained in the field grown selfed progeny of an autotriploid $H$. muticus generated from a cross between autotetraploid $\times$ diploid (inbred CIMAP/NP-41). For meiotic studies, floral buds were fixed in freshly prepared acetic-alcohol $(1: 3)$ solution and anthers squashed in $2 \%$ acetocarmine. Chromosome associations at diakinesis and/or metaphase I (MI) and their distributions at anaphase I (AI) and anaphase II (AII) were recorded.

Pollen fertility was determined by the percentage of stainable pollen with $1 \%$ acetocarmine. A minimum of 500 pollen grains were counted at random from each plant. The number of healthy seeds set in 20 capsules in each of the tetrasomics and normal diploid counterpart resulted from their self pollination were counted from which the seed set per capsule was calculated. The data on various morphological characters (at midflowering stage of plant growth) were recorded on both the tetrasomics and their standard diploid counterpart (CIMAP/ NP-41). Leaf area was measured using the area meter (LICOR-Model 3100).

\section{Results and discussion}

Addition or deletion of chromosomes to the diploid constitution provide interesting 


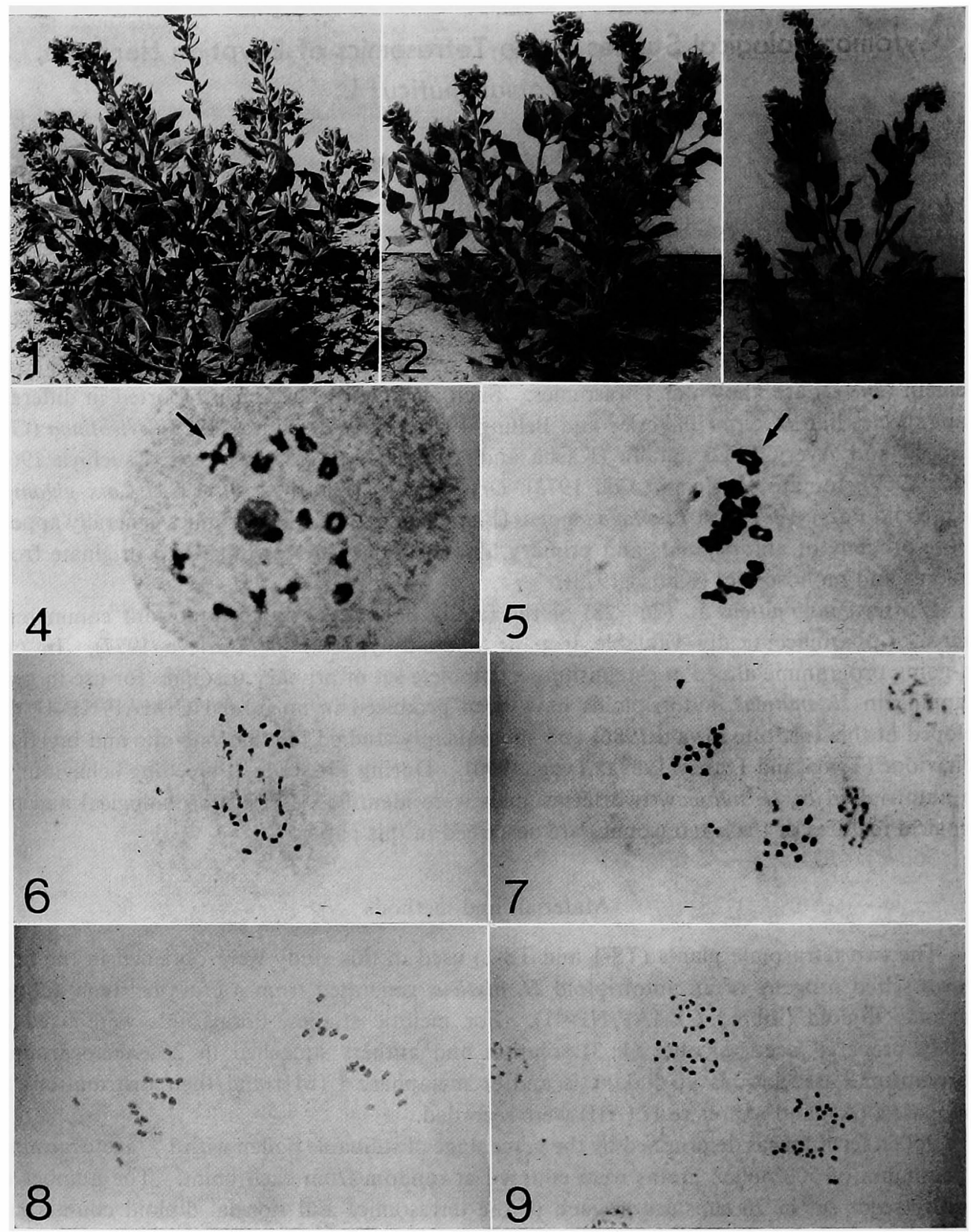

Figs. 1-9. Comparison of plant morphology of diploid and tetrasomics, different chromosome associations at diakinesis and metaphase I and their distribution at anaphase I and II in tetrasomic plants of $H$. muticus. $\quad$, a diploid plant of inbred CIMAP/NP-41. 2 and 3, a tetrasomic plant TS-1 and TS-2, respectively. 4, diakinesis with IIV (Chain)+13II. 5, metaphase I with 1IV (ring)+ 13II. 6, early anaphase I with $15: 15$ chromosome distribution. 7, anaphase I with $14: 15+1 \mathrm{~L}$ chromosome distribution. 8, metaphase II showimng 16 and 14 chromosomes lying on metaphase plates. 9, anaphase II with 16:14::13:17 chromosome distribution. Arrow(s) shows a quadrivalent $(\times 1000)$. 
information on cytogenetics and evolution. Tetrasomics occur comaparatively rarely on account of their infrequent origin and low survival, although these have been reported in some plant species. Generally, tetrasomics were obtained in the progeny of autotriploids and primary trisomics (Kasha and McLennan 1967, Rick and Gill 1973, Khush et al. 1984, Sapre and Barve 1985, 1987, Bhan et al. 1990), they are also likely to originate from disomics and monosomics (Khush 1973). In view of our earlier observations of only female transmission of extra chromosomes in $H$. muticus (Tyagi 1990), the occurrence of two tetrasomics TS-1 and TS-2 in the selfed progeny of autotriploid in the present case might be a result of fertilisation of female gamete having 16 chromosomes (which could have formed as a result of movement of all the three doses of one chromosome and single dose of the remaining chromosomes towards the same pole during chromosome disjunction) with a normal pollen. Although Tyagi (1990) has reported the maximum limit of tolerance of 4 extra chromosomes (quadruple primary trisomic) in $H$. muticus, the occurrence of tetrasomics as reported in this study suggests the plants with 4 doses of one chromosome (tetrasomy) can also be viable in $H$. muticus.

Table 1. Chromosome associations at diakinesis/metaphase-I in two tetrasomics of $H$. muticus

\begin{tabular}{|c|c|c|}
\hline \multirow{2}{*}{ Chromosome associations } & \multicolumn{2}{|c|}{ Ferquency $(\%)$} \\
\hline & TS-1 & TS-2 \\
\hline $1 \mathrm{IV}+13 \mathrm{II}$ & 62.29 & 34.52 \\
\hline $1 I I I+13 I I+1 I$ & 9.84 & 13.10 \\
\hline 1511 & 21.31 & 47.62 \\
\hline $14 I I+2 I$ & 4.10 & 3.57 \\
\hline $13 \mathrm{II}+4 \mathrm{I}$ & 2.46 & 1.19 \\
\hline Total number of cells & 244 & 168 \\
\hline
\end{tabular}

Table 2. Mean frequencies of chromosome associations in two tetrasomics of H. muticus

\begin{tabular}{ccccc}
\hline \hline \multirow{2}{*}{ Tetrasomic } & \multicolumn{4}{c}{ Chromosome association/cell } \\
\cline { 2 - 5 } & IV & III & II & I \\
\hline TS-1 & 0.62 & 0.10 & 13.47 & 0.28 \\
TS-2 & 0.35 & 0.13 & 13.99 & 0.25 \\
\hline Mean & 0.48 & 0.11 & 13.73 & 0.27 \\
\hline
\end{tabular}

In both the tetrasomics, the two extra chromosomes paired with the corresponding homologues and formed a quadrivalent in $62.3 \%$ and $34.5 \%$ of PMCs at diakinesis/MI in TS-1 and TS-2, respectively, thus revealing their tetrasomic nature. The quadrivalent either formed chain (Fig. 4) or ring (Fig. 5) at diakinesis and/or metaphase I. In none of the trisomics, the quadrivalent was associated with the nucleolus indicating that the chromosome 4 which is the nucleolar organising chromosome in H. muticus (Tyagi and Dubey 1989b, Tyagi and Gill 1990 ) is not involved in tetrasomy. Other associations like $2 \mathrm{IIs}, \mathrm{II}+2 \mathrm{Is}, \mathrm{III}+\mathrm{I}$ and rarely 4Is were also observed at diakinesis/MI, depending upon the degree of synapsis, and number and position of chiasma(ta) among four homologues, though in decreasing order in both the tetrasomics (Table 1). The mean frequencies of chromosome associations observed were: $0.48 \mathrm{IV}, 0.11 \mathrm{III}, 13.73 \mathrm{II}$ and 0.27 I (Table 2). Similar observations have also been earlier reported in Coix gigantea (Sapre and Barve 1987) and Plantago lagopus (Bhan et al. 1990). A new type of tetrasomic plant in barley reported by Tsuchiya $(1962,1969)$ needs special mention here. As this plant carried extra chromosome 6 which showed structural changes, a 
pericentric inversion and a deletion. None of the PMCs showed presence of quadrivalent or trivalent because of the changed morphology of additional pair of chromosomes indicating how homologous chromosomes can behave as non homologous with the induction of structural changes. A tetratrisomic $(2 n+2+1)$ plant of Pennisetum americanum has also been isolated from among the progeny of open pollinated triploids (Sai Kumar et al. 1982). The three extra chromosomes are reported to produce quadrivalent and trivalent configurations proving its tetra-trisomic nature.

The cytological data on segregation of chromosomes at AI and AII summarised in Table 3 suggest that majority of the PMCs showed regular distribution of 15:15 chromosomes at AI (Fig. 6) indicating 2-2 disjunction of quadrivalent and 3-1 disjunction in some PMCs resulting into 14: 16 chromosome distribution (Fig. 8). Laggards ranging from 1 to 3 have been observed at AI. In most of the laggards at AI the chromatids separated precociously (Fig. 7), such a behaviour of laggards has also been reported earlier in H. muticus (Tyagi and Dubey 1989a) and Solanum (Lange and Wagenvoort 1973). The observed segregation of chromosomes at AI suggests how the meiotic products with $n-1$ to $n+3$ are likely to be formed

Table 3. Chromosome distribution at anaphase I and anaphase II in two tetrasomics of $H$. muticus

\begin{tabular}{crc}
\hline \multirow{2}{*}{$\begin{array}{c}\text { Chromosome } \\
\text { distribution }\end{array}$} & \multicolumn{2}{c}{ Frequency (\%) } \\
\cline { 2 - 3 } & TS-1 & TS-2 \\
\hline Anaphase I & $(113)$ & $(73)$ \\
$15: 15$ & 32.74 & 49.32 \\
$15: 14+1 \mathrm{~L}$ & 26.55 & 21.92 \\
$14: 14+2 \mathrm{~L}$ & 15.04 & 19.18 \\
$14: 13+3 \mathrm{~L}$ & 11.50 & 2.74 \\
$14: 16$ & 7.96 & 6.85 \\
$13: 17$ & 3.54 & - \\
Anaphase 11 & $(28)$ & $(32)$ \\
$15: 15:: 15: 15$ & 32.14 & 43.75 \\
$16: 16:: 14: 14$ & 39.28 & 18.75 \\
$16: 14:: 13: 17$ & 10.71 & - \\
$14: 14:: 15: 15+2 \mathrm{~L}$ & 3.57 & 21.87 \\
$14: 14:: 14: 15+3 \mathrm{~L}$ & 14.29 & 15.63 \\
\hline
\end{tabular}

Figures in parenthesis represent the number of PMCs observed.

in the two tetrasomics under study. Failure of quadrivalent to disjoin and its passing towards the same pole producing $n+3(n=17)$ and $n-1(n=13)$ gametes is indicated by the occurrence of 13:17 distribution of chromosomes at $\mathrm{AI}$ in 3.54\% PMCs in tetrasomic TS-1.

The comparative data on morphological features, pollen fertility and seed set of both the tetrasomics (TS-1 and TS-2, Figs. 2 and 3, respectively) alongwith normal diploid (CIMAP/ NP-41, Fig. 1) are summarised in Table 4. The overall morphology of tetrasomics was similar to that of diploid counterpart CIMAP/NP-41 except reduction in plant height and number of primary branches, and increase in average leaf area. Furthermore, a considerable decrease in pollen fertility and seed set per capsule was observed in both the tetrasomics which is about $40-60 \%$ and $12-18 \%$, respectively of the diploid. This might be a result of disturbed gene balance caused by the presence of extra doses of homologous chromosomes. Tetrasomic TS-1 resembled the diploid in appearance. However, it may be readily distinguished from the diploid and tetrasomic TS-2 by its broad and dark green leaves. Whereas, tetrasomic TS-2 had thick glossy leaves with almost the same leaf area as that of diploid. These observed differences in morphology, meiotic behaviour, pollen fertility and seed set in the two tetrasomics 
indicate that two different chromosomes are involved in these tetrasomics. Similar morphological changes like stunted growth and high sterlity associated with tetrasomy have been reported earlier in tomato (Rick and Gill 1973) However, tetrasomic plants in barley (Tsuchiya 1969) and Plantago lagopus (Bhan et al. 1990) were reported to have high pollen fertility with no marked morphological differences.

The progeny of tetrasomics mostly consist of disomics. trisomics and tetrasomics on account of 3-1 and 2-2 disjunction of quadrivalent as revealed from earlier breeding experiments conducted on tetrasomics of Datura (Blakeslee and Avery 1938) and wheat (Sears 1954). The fertility, though low in these tetrasomics of $H$. muticus seems to be adequate for their utilisation in isolating two different primary trisomics.

Table 4. Morphological features, pollen fertility and seed set in diploid and tetrasomic plants of $H$. muticus

\begin{tabular}{|c|c|c|c|c|}
\hline \multirow{2}{*}{ S1. No. } & \multirow{2}{*}{ Characters } & \multirow{2}{*}{ Diploid $(2 n)$} & \multicolumn{2}{|c|}{ Tetrasomic $(2 n+2)$} \\
\hline & & & TS-1 & TS-2 \\
\hline 1. & Plant height $(\mathrm{cm})$ & $\begin{array}{c}73.64 \\
(66.9-80.5)\end{array}$ & 64.60 & 62.90 \\
\hline 2. & Primary branches/plant & $\begin{array}{r}12.2 \\
(10-15)\end{array}$ & 5 & 2 \\
\hline 3. & Girth of main shoot $(\mathrm{cm})$ & $\begin{array}{r}5.68 \\
(3.2-8.1)\end{array}$ & 5.80 & 5.60 \\
\hline 4. & Leaf area/leat $\left(\mathrm{cm}^{2}\right)$ & $\begin{array}{c}71.98 \\
(18.6-156.2)\end{array}$ & $\begin{array}{c}81,64 \\
(24.6-180.2)\end{array}$ & $\begin{array}{c}76.42 \\
(19.4-162.4)\end{array}$ \\
\hline 5 & Flower length $(\mathrm{mm})$ & $\begin{array}{c}30.10 \\
(17-41)\end{array}$ & $\begin{array}{r}33.80 \\
(19-48)\end{array}$ & $\begin{array}{c}30.80 \\
(16-42)\end{array}$ \\
\hline 6. & Flower width (mm) & $\begin{array}{r}20.40 \\
(16-27)\end{array}$ & $\begin{array}{r}21.00 \\
(17-29)\end{array}$ & $\begin{array}{r}20.20 \\
(16-28)\end{array}$ \\
\hline 7. & Anther length (mm) & $\begin{array}{l}4.80 \\
(4-6)\end{array}$ & $\begin{array}{l}3.80 \\
(3-5)\end{array}$ & $\begin{array}{l}5.40 \\
(4-7)\end{array}$ \\
\hline 8. & Style length (mm) & $\begin{array}{c}19.40 \\
(17-23)\end{array}$ & $\begin{array}{c}16.40 \\
(14-20)\end{array}$ & $\begin{array}{c}19.80 \\
(17-24)\end{array}$ \\
\hline 9. & Size of pollen grains $\left(\mu \mathrm{m}^{2}\right)$ & $\begin{array}{c}1188.56 \\
(916-1420)\end{array}$ & $\begin{array}{c}1256.76 \\
(817-1568)\end{array}$ & $\begin{array}{c}1163.08 \\
(951-1388)\end{array}$ \\
\hline 10 & Pollen fertility $(\%)$ & $\begin{array}{c}96.48 \\
(92.3-99.6)\end{array}$ & 34.80 & 53.70 \\
\hline 11. & Seeds/capsule & $\begin{array}{c}247.4 \\
(178-313)\end{array}$ & $\begin{array}{r}29.2 \\
(15-51)\end{array}$ & $\begin{array}{r}43.2 \\
(31-58)\end{array}$ \\
\hline
\end{tabular}

Figures in parenthesis represent range.

\section{Summary}

Cytomorphological studies on $H$. muticus tetrasomics $(2 n+2=30)$, isolated from among the selfed progeny of autotriploids, and their diploid $(2 n=28)$ counterpart inbred CIMAP/ NP-41 were carried out. The overall morphology of both the tetrasomics was similar to that of diploid except reduction in plant height, number of primary branches, pollen fertility and seed set per capsule and increase in leaf area per leaf.

The four homologous chromosomes formed either a quadrivalent or two bivalents at diakinesis/metaphase I in majority of the PMCs in both the tetrasomics. Other associations like II $+2 \mathrm{Is}$, III $+\mathrm{I}$ and rarely $4 \mathrm{Is}$ were also observed in decreasing order. The mean frequencies of chromosome associations in terasomics were $0.48 \mathrm{IV}, 0.11 \mathrm{III}, 13.73 \mathrm{II}$ and $0.27 \mathrm{I}$. Gametes with variable number of chromosomes ranging between $n-1$ and $n+3$ were produced as a result of 3-1 and 2-2 disjunction of quadrivalent. The differences in morphological 
features, meiotic behaviour, pollen fertility and seed set in the two tetrasomics suggested the involvement of different chromosomes in tetrasomy.

\section{References}

Bhan, A., Koul, A. K. and Langer, A. 1990. Genetic diversity among Plantagos-XXII. Tetrasomy in Plantago lagopus L. Nucleus 33: 134-137.

Blakeslee, A F. and Avery, A. G. 1938. Fifteen-year breeding records of $2 n+1$ types in Datura stramonium. Coop. in Res. Carnegie Inst. Wash. Pub. 501: 315-351.

— and Belling, J. 1924. Chromosomal mutations in Jimson Weed, Datura stramonium. J. Hered. 15: 195-206.

Goodspeed, T. H. and Avery, P. 1939. Trisomics and other types in Nicotiana sylvestris. J. Genet. 36: 381-458.

Grieve, M. 1977. Henbane. In A Modern Herbal. ed. C. F. Leyel. Jonathan Cape, London, pp. 397-404.

Kasha, K. J. and McLennan, H. A. 1967. Trisomics in diploid alfalfa. I. Production, fertility and transmission. Chromosoma 21: 232-242.

Khush, G. S. 1973. Cytogenetics of Aneuploids. Acad. Press, New York and London, 301 pp.

-, Singh, R. J., Sur, S. C. and Librojo, A. L. 1984. Primary trisomics of rice: Origin, morphology, cytology and use in linkage mapping. Genetics 107: 141-163.

Lange, W. and Wangenvoort, M. 1973. Meiosis in triploid Solanum tuberosum L. Euphytica 22: 8-18.

McClintock, B. 1929. A cytological and genetical study of triploid maize. Genetics 14: 180-222.

Rick, C. M. and Gill, B. S. 1973. Reproductive errors in aneuploids: Generation of variant extra-chromosomal types by tomato primary trisomics. Can. J. Genet. Cytol. 15: 299-308.

Sai Kumar, R., Singh, U. P., Singh, R. B. and Singh, R. M. 1982. Cytological behaviour of a tetra-trisomic plant in pearl millet (Pennisetum americanum (L.) K. Schum). Curr. Sci. 51: 376-378.

Sapre, A. B. and Barve, S. S. 1985. Cytology of higher polysomics in Coix gigantea (Poaceae): Pentasomy and hexasomy. Genetica 66: 73-80.

- and - 1987. Cytological studies in the tetrasomics of Coix gigantea (Poaceae). Cytologia 52: 323-330.

Sears, E. R. 1954. The aneuploids of common wheat. M. Agr. Exp. Stn. Res. Bull. No. 572, 58 pp.

Tsuchiya, T. 1962. A tetrasomic plant in barley. Chrom. Inf. Serv. 3: 17-18.

- 1967. Establishment of a trisomic series in a two rowed cultivated variety of barley. Can. J. Genet. Cytol. 9: $667-682$.

- 1969. Status of studies of primary trisomics and other aneuploids in barley. Genetica 40: 216-232.

Tyagi, B. R. 1986. Development of higher tropane alkaloids containing stable genotypes in Egyptian henbane (Hyoscyamus muticus L.). In Plantation Crops-Opportunities and constraints. eds. H. C. Srivastava, B. Vatsya and K. K. G. Menon. Oxford and IBH Publishing Co., New Delhi, Vol. I, pp. 387-390.

- 1990. Breeding behaviour of an autotriploid in Hyoscyamus muticus L. Cytologia 55: 153-159.

- and Dubey, R. 1989a. Meiotic chromosome behaviour and fertility in autotriploids of Egyptian henbane, Hyoscyamus muticus L. Caryologia 42: 153-163.

- and - 1989b. Karyotype analysis of Egyptian henbane, Hyoscyamus muticus L. Cytologia 54: 313-317.

— and Gill, B. S. 1990 . C-banded karyotype of Hyoscyamus muticus L. J. Hered. 81 : 401-402. 